\title{
Safety of Protected Carotid Artery Stenting in Patients with Severe Carotid Artery Stenosis and Carotid Intraplaque Hemorrhage
}

W. Yoon BACKGRound AND PURPOSE: Carotid IPH can be detected with MR imaging. The aim of this study S.K. Kim was to determine the safety of CAS using an emboli protection device in patients with severe carotid M.S. Park H.J. Chae H.K. Kang artery stenosis and MR imaging-depicted carotid IPH.

MATERIALS AND METHODS: We retrospectively reviewed a prospective data base that included 91 consecutive patients with severe carotid stenosis and high-risk features who were treated with CAS by using an emboli protection device. Seventy-eight of the included patients underwent prestenting 3D TOF MRA. IPH was defined as the presence of high signal intensity within the carotid plaque, greater than $150 \%$ of the signal intensity of the adjacent neck muscle on TOF source images. The primary outcome measure was the combined incidence of stroke, MI, and death within 30 days of CAS. Associations between IPH and the primary outcome were investigated.

RESULTS: IPH was detected on TOF MRA in 30 patients. Symptomatic patients were more common in the IPH group than in the non-IPH group $(66.7 \%$ vs $41.7 \% ; P=.032)$. Overall, 30-day stroke, $\mathrm{MI}$, or death rates were $6.6 \%$. There was no significant difference in the primary outcome between the IPH and non-IPH groups $(10 \%$ and $6.25 \%$, respectively; hazard ratio for $\mathrm{IPH}, 1.151 ; 95 \% \mathrm{Cl}, 0.035$ to 37.500; $P=.937)$. A logistic regression showed there was no independent variable associated with the primary outcome.

CONCLUSIONS: The results of this study indicate that protected CAS seems to be safe in patients with severe carotid stenosis and IPH.

ABBREVIATIONS: CAS = carotid artery stenting; CEA = carotid endarterectomy; $\mathrm{Cl}=$ confidence interval; CREST = Carotid Revascularization Endarterectomy vs Stenting Trial; IPH = intraplaque hemorrhage; $\mathrm{MI}=$ myocardial infarction; MPRAGE = magnetization-prepared rapid acquisition with gradient echo; TOF = time-of-flight

A therosclerotic carotid artery stenosis is an important cause of ischemic stroke, accounting for approximately $20 \%$ of all ischemic strokes. ${ }^{1}$ CEA has been established as an effective treatment for both symptomatic and asymptomatic patients. ${ }^{2-4}$ CAS is an alternative approach for treating carotid stenosis in high surgical risk patients, particularly those with symptomatic high-grade stenosis. The results of the CREST study showed that outcomes with CAS are similar to those with CEA in both the short and long term. ${ }^{5}$

IPH is a critical factor in the destabilization and growth of carotid atherosclerotic plaques, ${ }^{6,7}$ and is associated with the development of future cerebral ischemic events. ${ }^{8-10} \mathrm{MR}$ imaging is a valuable noninvasive tool for characterizing carotid atherosclerotic plaques. Carotid IPH can be identified in T1-

Received July 8, 2011; accepted after revision September 12, 2011.

From the Departments of Radiology (W.Y., S.K.K., H.K.K.), Neurology (M.S.P.), and Occupational and Environmental Medicine (H.J.C.), Chonnam National University Medical School, Chonnam National University Hospital, Gwangju, Republic of Korea.

This work was supported in part by a research grant from GE Healthcare. This GEHC Research Grant Program is a competitive grant program for radiologists in South Korea.

Paper previously presented as an oral presentation at: Annual Meeting of the American Society of Neuroradiology, June 7, 2011; Seattle, Washington.

Please address correspondence to Dr. Woong Yoon, Department of Radiology, Chonnam National University Hospital, 671 Jebong-Ro, Dong-gu, Gwangju, 501-757, Republic of Korea; e-mail: radyoon@jnu.ac.kr

Indicates open access to non-subscribers at www.ajnr.org

http://dx.doi.org/10.3174/ajnr.A2911 weighted MR imaging sequences. ${ }^{11-13}$ The presence of IPH at high risk of causing thromboembolic events may increase the risk of distal embolization during the carotid revascularization procedures. However, no studies investigating the safety of CAS in patients with carotid IPH have been previously conducted. Therefore, the present study was performed with the goal of assessing the safety of CAS using an emboli protection device in patients with high-grade carotid stenosis and carotid IPH.

\section{Materials and Methods}

\section{Study Population}

From a prospective registry of 91 consecutive patients who were treated with elective CAS and distal embolic protection, we selected 80 patients who underwent neck 3D TOF MRA within 1 month before CAS. Two patients whose neck MRA was of insufficient quality for reliable evaluation were excluded, leaving 78 patients in the study. This study was a retrospective analysis approved by our institutional review board. Informed consent for the retrospective analysis of the patient data was waived by the institutional review board.

According to previously published criteria, ${ }^{14}$ all 78 patients undergoing protected CAS are at high surgical risk for carotid endarterectomy. The high-risk features included were high cervical carotid stenosis $(n=38)$, clinically significant cardiac disease $(n=12)$, contralateral carotid occlusion $(n=10)$, age $\geq 80$ years $(n=8)$, tandem stenosis $\geq 70 \%(n=6)$, and severe pulmonary disease $(n=4)$. All patients had severe carotid stenosis (70\% to $99 \%$, based on NASCET criteria) on conventional angiography. 
To evaluate baseline characteristics of the patients, the risk factors considered were defined as follows: history of ischemic heart disease was defined as a previous MI or coronary artery bypass grafting; dyslipidemia was defined as hypercholesterolemia (total cholesterol $>220 \mathrm{mg} / \mathrm{dL}$, low-attenuation lipoprotein $>160 \mathrm{mg} / \mathrm{dL}$, triglyceride $>150 \mathrm{mg} / \mathrm{dL}$, or high-attenuation lipoprotein $<40 \mathrm{mg} / \mathrm{dL}$ ) or treatment with cholesterol-lowering medication; hypertension was defined as systolic blood pressure $>140 \mathrm{~mm} \mathrm{Hg}$ and/or a diastolic blood pressure $>90 \mathrm{~mm} \mathrm{Hg}$, or treatment with antihypertensive medication; diabetes mellitus was defined as a fasting serum glucose level $>110 \mathrm{mg} / \mathrm{dL}, \mathrm{HbAlc}>6.5 \%$, or use of antidiabetic medication.

Symptomatic carotid artery stenosis was defined as the onset of focal neurologic symptoms (TIA or nonfatal stroke) occurring within 6 months of CAS and attributable to an ipsilateral carotid artery vascular distribution.

\section{Carotid Stent Placement Procedure}

Between August 2006 and June 2010, 91 consecutive patients with severe carotid stenosis and high-risk features were treated with protected CAS at our institution. The CAS procedures were performed by 1 interventional neuroradiologist with 10 years' experience in neurovascular intervention (W.Y.). Written informed consent for the CAS procedure was obtained from all patients. All patients were examined by a stroke neurologist before and after the procedure to document cerebrovascular symptom status and to record any new neurologic deficits. All patients were treated with aspirin at a dose of $100 \mathrm{mg} /$ day and clopidogrel at a dose of $75 \mathrm{mg} /$ day for at least 3 days before the procedure. At the beginning of the procedure, heparin was given intravenously as a $3000 \mathrm{IU}$ bolus. All patients received aspirin and clopidogrel for at least 3 months after CAS.

All procedures were performed using a femoral approach. After placement of the $8 \mathrm{~F}$ guiding catheter, diagnostic common carotid angiography was performed to determine the severity of carotid stenosis according to NASCET criteria. CAS was performed with the SpiderRX distal embolic protection system (ev3, Plymouth, Minnesota). After crossing the lesion with a microguidewire, the filter was deployed before predilation was performed. Predilation was performed with a 5- or 6-mm balloon catheter. CAS was completed using a RX Acculink carotid stent (Abbott Vascular, Santa Clara, California). Postdilation was not routinely performed. After CAS was completed, the filter was captured and retrieved by the delivery catheter, followed by completion of the angiography. Procedural success was defined as completion angiography showing $<30 \%$ residual stenosis, without alteration in the intracranial circulation. After the procedure, all patients were monitored in the intensive care unit for 24 hours.

\section{MR Imaging Examinations and Image Analysis}

MR imaging examinations were performed on a $1.5 \mathrm{MR}$ imaging scanner (Signa HDxt; GE Healthcare, Milwaukee, Wisconsin) using an 8-channel phased-array coil, and included routine clinical brain MR imaging sequences, 3D TOF MRA of the circle of Willis, and neck 3D TOF MRA. The indications for MR imaging examinations were TIA or ischemic stroke $(n=40)$, headache $(n=22)$, routine medical work-up $(n=14)$, and amaurosis fugax $(n=2)$. The mean period between MR imaging and CAS was 16 days, ranging from 1-30 days.

The neck 3D TOF MRA was acquired with a multiple overlapping thin-slab acquisition in the axial plane using a gradient-echo technique with the following parameters: TR/TE/flip angle, $24 \mathrm{~ms} / 6.8$ $\mathrm{ms} / 20^{\circ}$; FOV, $240 \times 240 \mathrm{~mm}^{2}$; matrix, $384 \times 160$; section thickness, $2 \mathrm{~mm}$; 65 sections. The acquired voxel size was $0.63 \times 1.5 \times 2 \mathrm{~mm}^{3}$ and was reconstructed to a matrix of $512 \times 512$, with interpolation to 132 sections at $1 \mathrm{~mm}$. The total coverage was $130 \mathrm{~mm}$, and the scan time was 2 minutes 15 seconds.

Two neuroradiologists reviewed the TOF source images of the carotid bifurcation and the proximal internal carotid artery, and final interpretations were reached by consensus. For interpretation of the MRA, these radiologists were blinded to patient clinical information and findings from the conventional angiography. Only axial source images of the TOF MRA were used for image evaluation. All images were reviewed on a PACS system. For analysis, MR imaging signal intensities were measured in a 6 - to $10-\mathrm{mm}^{2}$ circular region of interest over the carotid plaque. IPH was defined as the presence of a hyperintense signal intensity within the carotid plaque greater than $150 \%$ of the signal intensity of the adjacent neck muscle on TOF source images. ${ }^{8}$

\section{Outcome}

Neurologic evaluation was performed at baseline, immediately and 24 hours after the procedure, at the time of any change in clinical symptoms, before patient discharge, and 1 month afterward by a stroke neurologist. The evaluation consisted of the NIHSS and the modified Rankin Scale.

The primary outcome measure was the combined incidence of any stroke, MI, or death during the 30-day periprocedural period. Stroke was defined as an acute neurologic event with focal symptoms and signs, lasting for 24 hours or more, that were consistent with focal cerebral ischemia. A major stroke was defined as stroke symptoms associated with a NIHSS score of 9 or higher 30 days after the procedure. A minor stroke was defined as stroke symptoms associated with a NIHSS score of 8 or less. MI was defined as the combination of elevated cardiac enzymes (creatinine kinase-MB or troponin level) to a value 2 or more times the upper limit of normal, plus chest pain.

The secondary outcome measures were as follows: 1) technical success, defined as successful deployment of all devices, filter retrieval, and poststent diameter stenosis $<30 \%$ using the NASCET method; 2) hyperperfusion syndrome, defined as the occurrence, either singly or in combination, of an ipsilateral throbbing headache with or without nausea, vomiting, ipsilateral focal seizures, or focal neurologic deficit without radiographic evidence of infarction ${ }^{15}$; and 3) ICH associated with hyperperfusion syndrome, defined as CT evidence of intraparenchymal or SAH accompanied by hyperperfusion syndrome. The patients were assessed clinically at the follow-up visit 30 days after CAS. Follow-up CT angiography or conventional angiography was not performed within 30 days.

\section{Statistical Analysis}

Patients were divided into the IPH group or the non-IPH group for comparison. Statistical analyses were performed with SPSS software (Version 15.0; SPSS, Chicago, Illinois). Comparison of baseline characteristics and clinical outcomes between the 2 groups was done with the $\chi$-square test for categoric and binary data, and the Student $t$ test for continuous data. Multiple logistic regression was run with the dependent variable as a primary outcome. The independent variables were IPH, age, sex, index event, cerebrovascular history, history of ischemic heart disease, and cerebrovascular risk factors. A value of $P<.05$ was considered significant.

\section{Results}

\section{Patient Demographics}

Data from 78 patients were analyzed. Most patients were male $(n=69 ; 88.5 \%)$ and the mean age was $70.8 \pm 5.67$ years. TOF 

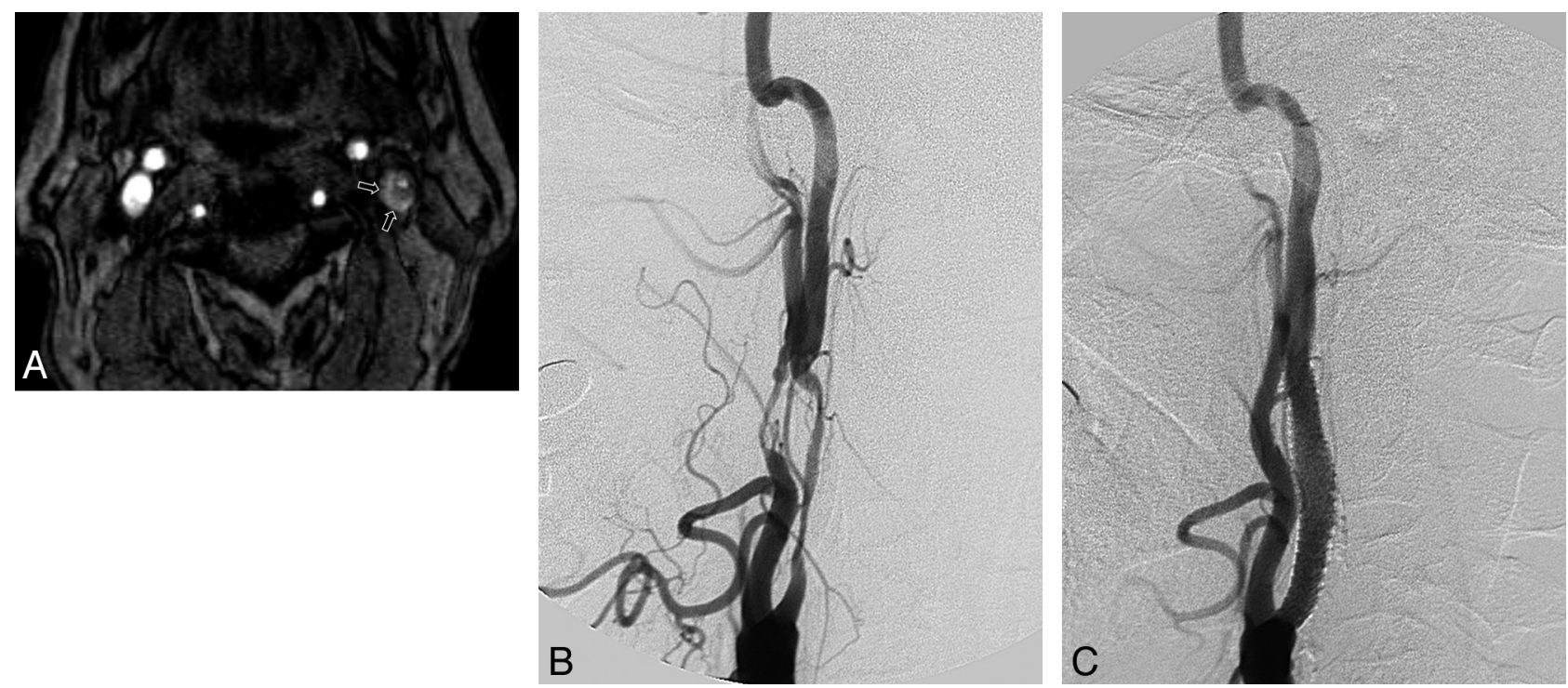

Fig 1. An example of intraplaque hemorrhage seen on a TOF source image in a 71-year-old man with a left cerebral ischemic event. $A$, Axial source image of TOF MRA shows the IPH (short arrows) in the eccentric left carotid plaque, which surrounds the lumen of the internal carotid artery with severe stenosis. IPH is identified as a hyperintense signal intensity compared with the adjacent neck muscle. $B$, Lateral projection of a left carotid angiogram shows long segmental severe stenosis in the proximal cervical portion of the left internal carotid artery. $C$, Postcarotid artery stent placement angiogram shows minimal residual stenosis at the origin site of the left internal carotid artery.

\begin{tabular}{lccc}
\hline \multicolumn{4}{l}{ Table 1: Baseline characteristics of the study population } \\
\hline & $\begin{array}{c}\text { No IPH } \\
(n=48 ;\end{array}$ & $\begin{array}{c}\text { IPH } \\
(n=30 ;\end{array}$ & \\
& $61.5 \%)$ & $38.5 \%)$ & $P$ \\
\hline Age (years) & $71 \pm 7$ & $70 \pm 7$ & 0.880 \\
Sex, male & $41(85.4 \%)$ & $28(93.3 \%)$ & 0.469 \\
Index event & & \\
TIA & $8(16.7 \%)$ & $4(13.3 \%)$ & 0.758 \\
Ischemic stroke & $12(25 \%)$ & $16(53.3 \%)$ & 0.011 \\
TIA or ischemic stroke & $20(41.7 \%)$ & $20(66.7 \%)$ & 0.032 \\
Cerebrovascular history & & & \\
Previous ischemic stroke & $11(22.9 \%)$ & $7(23.3 \%)$ & 0.966 \\
History of ischemic heart disease & $13(27.1 \%)$ & $8(26.7 \%)$ & 0.968 \\
Cerebrovascular risk factors & & & \\
Hypertension & $34(70.8 \%)$ & $24(80 \%)$ & 0.367 \\
Diabetes mellitus & $21(43.8 \%)$ & $12(40 \%)$ & 0.744 \\
Dyslipidemia & $9(18.8 \%)$ & $10(33.3 \%)$ & 0.144 \\
Atrial fibrillation & $6(12.5 \%)$ & $1(3.3 \%)$ & 0.240 \\
Smoking & $24(50 \%)$ & $17(56.7 \%)$ & 0.566 \\
\hline
\end{tabular}

Note:-Data are means \pm SD or number of patients $(\%)$.

source images demonstrated IPH in 30 patients (38.5\%) (Fig 1 ). The baseline characteristics of patients with and without IPH are presented in Table 1. Overall, 28 patients presented with nonfatal ischemic stroke before CAS, and 12 presented with TIA. Nonfatal ischemic stroke as an index event was significantly more common in the IPH group (53.3\%) than in the non-IPH group $(25 \% ; P=.011)$. The prevalence of TIA before CAS was not different between the 2 groups. Overall, symptomatic patients were more common in the IPH group than in the non-IPH group ( $66.7 \%$ versus $41.7 \% ; P=.032)$. The other baseline characteristics were similar between the 2 groups.

\section{Outcome}

Overall, 30-day stroke, MI, or death rates were $6.6 \%$ (6 of 91 patients) during the study period. Four patients had a minor stroke, and none had a major stroke during the 30 days after CAS. The 30-day clinical outcomes of patients with and with-
Table 2: Thirty-day clinical outcomes after protected carotid artery stenting

\begin{tabular}{lccc}
\hline & $\begin{array}{c}\text { No IPH } \\
(n=48 ;\end{array}$ & $\begin{array}{c}\text { IPH } \\
(n=30 ;\end{array}$ & \\
& $61.5 \%)$ & $38.5 \%)$ & $P$ \\
\hline Hyperperfusion syndrome & $1(2.1 \%)$ & $2(6.7 \%)$ & 0.555 \\
Minor stroke & $2(4.2 \%)$ & $2(6.7 \%)$ & 0.636 \\
Major stroke & 0 & 0 & - \\
Ml & 0 & $1(3.3 \%)$ & 0.385 \\
Death & $1(2.1 \%)$ & $1(3.3 \%)$ & 1.000 \\
Stroke or Ml or death & $3(6.25 \%)$ & $3(10 \%)$ & 0.670 \\
\hline
\end{tabular}

Note:-Data are numbers of patients $(\%)$

out IPH are shown in Table 2. One patient with IPH died of an acute MI during a coronary stent placement procedure 2 days after the CAS. One patient in the non-IPH group died of an acute subarachnoid hemorrhage and hyperperfusion syndrome, which occurred 4 hours after the CAS. There were no mortalities related to ischemic complications.

There was no significant difference in the primary outcome between the IPH and non-IPH groups $(10 \%$ and $6.25 \%$, respectively; hazard ratio for IPH, $1.151 ; 95 \% \mathrm{CI}, 0.035$ to 37.500; $P=$.937). Multiple logistic regression showed that there was no other independent variable associated with the primary outcome by univariate and multivariate analysis, as well as IPH.

Technical success was achieved in all patients. Isolated hyperperfusion syndrome occurred in 1 patient $(1.1 \%)$, and ICH occurred in 2 patients $(2.2 \%)$, for a combined incidence of $3.3 \%$ in 91 CAS procedures. One patient with nonhemorrhagic hyperperfusion syndrome in the IPH group showed diffuse brain swelling in the ipsilateral cerebral hemisphere upon immediate poststenting CT, and had mild disability 30 days after CAS (NIHSS score $=4$ ). There was 1 death associated with intracranial hemorrhage and hyperperfusion syndrome in the non-IPH group, as described above. One patient with IPH showed localized SAH and ICH in the ipsilateral 
Table 3: Incidence of clinical outcome according to the presence or absence of patient symptoms before CAS

\begin{tabular}{lccccc}
\hline & \multicolumn{2}{c}{$\begin{array}{c}\text { Symptomatic } \\
(n=40 ; 51.7 \%)\end{array}$} & & \multicolumn{2}{c}{$\begin{array}{c}\text { Asymptomatic } \\
(n=38 ;\end{array}$} \\
\cline { 2 - 3 } \cline { 5 - 6 } & $\begin{array}{c}\text { IPH } \\
(n=20)\end{array}$ & $\begin{array}{c}\text { No IPH } \\
(n=20)\end{array}$ & & $\begin{array}{c}\text { IPH } \\
(n=10)\end{array}$ & $\begin{array}{c}\text { No IPH } \\
(n=28)\end{array}$ \\
\hline Hyperperfusion syndrome & 2 & 1 & & 0 & 0 \\
Minor stroke & 2 & 1 & & 0 & 1 \\
Major stroke & 0 & 0 & & 0 \\
Ml & 1 & 0 & & 0 \\
Death & 1 & 1 & & 0 & 0 \\
Stroke or Ml or death & 3 & 2 & & 0 & 1 \\
\hline
\end{tabular}

Note:-Data are numbers of patients.

temporal lobe upon immediate poststenting CT, but had no neurologic symptoms. He remained asymptomatic 30 days after CAS. There was no significant difference in the incidence of hyperperfusion syndrome between the IPH and non-IPH groups (Table 2).

The incidence of clinical outcomes, according to the presence or absence of patient symptoms before CAS, is presented in Table 3. Most (83\%) patients with primary outcome were symptomatic patients. The secondary outcome occurred exclusively in symptomatic patients.

\section{Discussion}

Our study indicates that protected CAS can be performed safely in patients with high-grade stenosis and carotid IPH. The findings of the current study showed that there was no statistically significant difference between the patients with IPH and those without in the combined incidence of stroke, MI, or death within 30 days of CAS. The results of the CREST study showed that stent placement and endarterectomy were equivalent in terms of the composite end points of stroke, MI, or death within 30 days, as well as for the rate of stroke for up to 4 years. ${ }^{5}$ CREST also showed that the risk of major stroke was low with either intervention $(0.9 \%$ for CAS and $0.6 \%$ for CEA). There was no major stroke in the current study, whether the patients did or did not have IPH. CREST reported a periprocedural minor stroke rate of $3.2 \%$. In our study, the overall minor stroke rate within 30 days was $4.4 \%$ (4 of 91).

Recent and fresh hemorrhage within the carotid plaque can be reliably identified with MR imaging using various T1weighted sequences such as black-blood spin-echo, gradientecho, TOF MRA, MPRAGE, and contrast-enhanced MRA. ${ }^{11-13}$ We used TOF MRA source images to detect carotid IPH in the present study. A high signal intensity area within the plaque around the carotid artery on TOF MRA, compared with histopathologic findings, demonstrated a sensitivity of $91 \%$ and a specificity of $83 \%$ in the depiction of an IPH. ${ }^{11}$

IPH plays a critical role in the evolution of carotid atherosclerotic disease. The presence of IPH in carotid atherosclerotic plaques has been associated with not only an accelerated growth of the plaque burden but also the development of future cerebral ischemic events. ${ }^{8-10}$ Several studies have shown that carotid IPH is also strongly associated with previous ipsilateral ischemic events and may predict subsequent cerebral ischemic events. ${ }^{10,16}$ The findings from our study are in agreement with previous studies. In the present study, IPH was more prevalent in symptomatic than in asymptomatic carotid plaques. Notably, nonfatal ipsilateral stroke as an index event was significantly more common in the IPH group than in the non-IPH group.

A recent study showed that there was increased spontaneous microembolic activity on transcranial Doppler imaging in patients with IPH shown on MR imaging scans. ${ }^{17}$ Thus, it can be postulated that a distal embolic event is more likely to occur during the CAS procedure in patients with IPH than in those without IPH. Yamada et al recently reported that the incidence of new ipsilateral silent DWI lesions was significantly greater after CAS than after CEA (61\% versus 13\%) in patients with carotid high-signal-intensity plaques, revealed by black-blood fast spin-echo T1-weighted sequences. ${ }^{18} \mathrm{~A}$ carotid highsignal-intensity plaque was defined as a signal intensity $\geq 125 \%$ that of the adjacent muscle in their study. They suggested that there might be a correlation between the carotid plaque instability and the incidence of distal embolism after CAS. However, they did not evaluate the associations between carotid high signal intensity plaques and the occurrence of ischemic symptoms after CAS. Besides intraplaque hemorrhage, lipid-rich necrotic core also can be attributed to high signal intensity on black-blood fast spin-echo T1-weighed sequences. ${ }^{19,20}$ In the current study, however, there was no statistically significant difference in the rates of minor or major stroke during the periprocedural period between IPH and non-IPH patients. This result can be attributed to the use of a distal emboli protection device, which may efficiently prevent the risk of distal embolism during a CAS procedure. We used a single type of distal embolic protection device in this study.

Sakamoto et al reported that carotid IPH can be used as a predictive factor of slow-flow phenomenon in CAS. ${ }^{21}$ In their study, slow-flow phenomena occurred in 10 of 31 CAS procedures, and 7 of 10 slow-flow phenomena were observed in patients with carotid IPH, revealed by multicontrast MR plaque imaging. We observed no slow-flow phenomena in this study.

There is only 1 report in the literature of a study of the association between carotid IPH and the risk of microembolization during CEA. ${ }^{22}$ Altaf et $\mathrm{al}^{17}$ found that carotid IPH, observed on MR imaging scans, is significantly associated with an increased risk of microembolization, detected on a transcranial Doppler imaging scan during the dissection phase of carotid endarterectomy. They suggested that the presence of IPH is a strong indicator of a carotid plaque that is likely to cause embolization during endarterectomy, and therefore the patient has a higher perioperative risk. ${ }^{17}$ In addition, Verhoeven et al showed that the number of microembolic events detected during the dissection phase of CEA is significantly related to immediate adverse neurologic events. ${ }^{23}$ Further studies are needed to clarify the appropriate treatment guidelines in patients with carotid stenosis and vulnerable plaque, such as IPH.

The limitations of this study were that the sample size was relatively small, and long-term follow-up data were not available. Longer follow-up is mandatory to draw more definitive conclusions about the safety of CAS in patients with vulnerable carotid plaque. Another limitation was that carotid IPH was determined with $1 \mathrm{MR}$ imaging sequence, TOF MRA, rather than a multicontrast protocol. A recent study indicated that the MPRAGE sequence, compared with the black-blood, 
fast spin-echo, and TOF sequences, demonstrated higher diagnostic capability for detecting and quantifying IPH. ${ }^{12}$ In addition, only IPH is considered a vulnerable characteristic of the carotid plaques in the current study. Although IPH contributes to plaque vulnerability, other factors such as a thin or ruptured fibrous cap, a large lipid-rich core, and wall thickness are also associated with subsequent cerebrovascular events. ${ }^{24}$ It would be useful to evaluate the association between other vulnerable characteristics of carotid plaques and the periprocedural safety of CAS in the future. Finally, the association of carotid IPH and cerebral ischemic symptoms remains somewhat controversial in the literature. There are various potential sources of heterogeneity and confounders among published studies addressing this issue regarding the definition of IPH; the criteria for determining age of IPH; size, number, and location of IPH; different degrees of carotid stenosis; and patient selection bias. A large well-designed study with uniformity in definition and evaluation for IPH might be warranted. ${ }^{25}$

\section{Conclusions}

We sought to assess the safety of protected CAS in patients with carotid IPH detected by MR imaging. We report that there was no significant difference in the 30-day clinical outcome between patients with IPH and those without IPH. The results of this study indicate that CAS using an emboli protection device seems to be safe in patients with high-grade carotid stenosis and IPH.

\section{References}

1. Chaturvedi S, Bruno A, Feasby T, et al. Carotid endarterectomy-an evidencebased review: report of the Therapeutics and Technology Assessment Subcommittee of the American Academy of Neurology. Neurology 2005;65:794801

2. North American Symptomatic Carotid Endarterectomy Trial Collaborators. Beneficial effect of carotid endarterectomy in symptomatic patients with high-grade carotid stenosis. N Engl J Med 1991;326:445-53

3. Halliday A, Mansfield A, Marro J, et al. Prevention of disabling and fatal strokes by successful carotid endarterectomy in patients without recent neurological symptoms: randomized control trial. Lancet 2004;363:1491-502

4. Adams RJ, Albers G, Alberts MJ, et al. Update to the AHA/ASA recommendations for the prevention of stroke in patients with stroke and transient attack. Stroke 2008;39:1647-52

5. Brott TG, Hobson RW 2nd, Howard G, et al. Stenting versus endarterectomy for treatment of carotid-artery stenosis. N Engl J Med 2010;363:11-23

6. Virmani R, Kolodgie FD, Burke AP, et al. Atherosclerotic plaque progression and vulnerability to rupture: angiogenesis as a source of intraplaque hemorrhage. Arterioscler Thromb Vasc Biol 2005;25:2054-61
7. Kolodgie FD, Gold HK, Burke AP, et al. Intraplaque hemorrhage and progression of coronary atheroma. N Engl J Med 2003;349:2316-25

8. Altaf N, MacSweeney ST, Gladman J, et al. Carotid intraplaque hemorrhage predicts recurrent symptoms in patients with high-grade carotid stenosis. Stroke 2007;38:1633-35

9. Hellings WE, Peeters W, Moll FL, et al. Composition of carotid atherosclerotic plaque is associated with cardiovascular outcome: a prognostic study. Circulation 2010;121:1941-50

10. Kurosaki Y, Yoshida K, Endo H, et al. Association between carotid atherosclerosis plaque with high signal intensity on T1-weighted imaging and subsequent ipsilateral ischemic events. Neurosurgery 2011;68:62-67

11. Yim YJ, Choe YH, Ko Y, et al. High signal intensity halo around the carotid artery on maximum intensity projection images of time-of-flight MR angiography: a new sign for intraplaque hemorrhage. J Magn Reson Imaging 2008;27:1341-46

12. Ota H, Yarnykh VL, Ferguson MS, et al. Carotid intraplaque hemorrhage imaging at 3.0-T MR imaging: comparison of the diagnostic performance of three T1-weighted sequences. Radiology 2010;254:551-63

13. Qiao Y, Etesami M, Malhotra S, et al. Identification of intraplaque hemorrhage on MR angiography images: a comparison of contrast-enhanced mask and time-of-flight techniques. AJNR Am J Neuroradiol 2011;32:454-59

14. Yadav JS, Wholey MH, Kuntz RE, et al. Protected carotid-artery stenting versus endarterectomy in high-risk patients. N Engl J Med 2004;351:1493-501

15. Abou-Chebl A, Yadav JS, Reginelli JP, et al. Intracranial hemorrhage and hyperperfusion syndrome following carotid artery stenting: risk factors, prevention, and treatment. J Am Coll Cardiol 2004;43:1596-601

16. Yamada N, Higashi M, Otsubo R, et al. Association between signal hyperintensity on T1-weighted MR imaging of carotid plaques and ipsilateral ischemic events. AJNR Am J Neuroradiol 2007;28:287-92

17. Altaf N, Goode SD, Beech A, et al. Plaque hemorrhage is a marker of thromboembolic activity in patients with symptomatic carotid disease. Radiology 2011;258:538-45

18. Yamada K, Yoshimura S, Kawasaki M, et al. Embolic complications after carotid artery stenting or carotid endarterectomy are associated with tissue characteristics of carotid plaques evaluated by magnetic resonance imaging. Atherosclerosis 2011;215:399-404

19. Yuan C, Mitsumori LM, Ferguson MS, et al. In vivo accuracy of multispectral magnetic resonance imaging for identifying lipid-rich necrotic cores and intraplaque hemorrhage in advanced human carotid plaques. Circulation 2001;104:2051-56

20. Cappendijk VC, Cleutjens KB, Heeneman S, et al. In vivo detection of hemorrhage in human atherosclerotic plaques with magnetic resonance imaging. $J$ Magn Reson Imaging 2004;20:105-10

21. Sakamoto $M$, Taoka $T$, Nakagawa $H$, et al. Magnetic resonance plaque imaging to predict the occurrence of the slow-flow phenomenon in carotid artery stenting procedures. Neuroradiology 2010;52:275-83

22. Altaf N, Beech A, Goode SD, et al. Carotid intraplaque hemorrhage detected by magnetic resonance imaging predicts embolization during carotid endarterectomy. J Vasc Surg 2007;46:31-36

23. Verhoeven BA, de Vries JP, Pasterkamp G, et al. Carotid atherosclerotic plaque characteristics are associated with microembolization during carotid endarterectomy and procedural outcome. Stroke 2005;36:1735-40

24. Gao T, Zhang Z, Yu W, et al. Atherosclerotic carotid vulnerable plaque and subsequent stroke: a high-resolution MRI study. Cerebrovasc Dis 2009;7: $345-52$

25. Gao P, Chen ZQ, Bao YH, et al. Correlation between carotid intraplaque hemorrhage and clinical symptoms: systematic review of observational studies. Stroke 2007;38:2382-90 